\title{
Template-Based Alignment Modeling of the Structure-Activity Relationships of CB2R Ligands
}

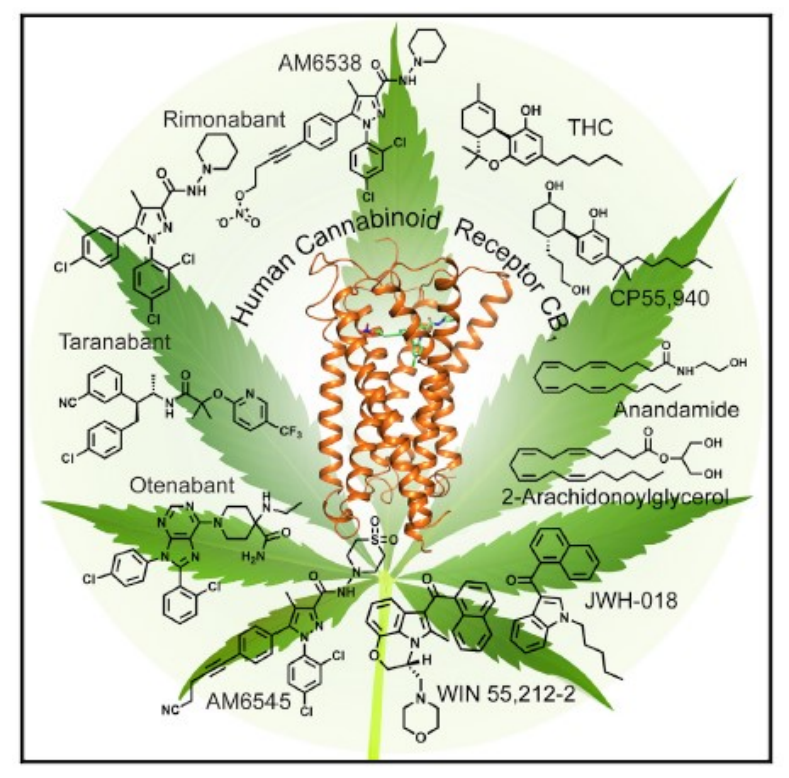

\author{
Zhijun Wu, PhD. \\ ABC Resource
}

ACS National Meeting \& Expo March 22 - 26, 2020 | Philadelphia, PA 


\section{Introduction}

Cannabinoid receptors (CBRs) are a type of G-protein-couple receptors (GPCRs) with two subtypes known, CB1R and CB2R. CB1R is mainly expressed in the central nervous system, while the $\mathrm{CB} 2 \mathrm{R}$ mainly in the immune system and in hematopoietic cells.

$\mathrm{CB} 2 \mathrm{R}$ is upregulated on microglial cells at the early stage of neurodegeneration, which makes CB2R a promising target for the early diagnosis and treatment of a variety of neurodegenerative disorders, including Alzheimer's disease, Parkinson disease, and others. To date a large number of CB2R ligands with diverse scaffolds have been reported.

Template-based alignment modeling (TAM) is an innovative SAR-modeling approach developed recently in our studies on opioid ligands. (Wu and Hruby, ACS Omega, Oct. 2019). In this study, by applying TAM, we investigated the structural correlations as well as the structure-activity relationships (SARs) of a variety of CBR ligands. Herein, we will first show how an artificial template of CB ligands was constructed. And we will then demonstrate how to utilize the template to understand the SARs of various CBR ligands with respect to their structural correlations and the structural features of CB2-selectivity as well as CB-ligand antagonism. 


\section{Cannabinoids}

There exist three major groups of CB ligands, including endocannabinoids, phytocannabinoids, and synthetic cannabinoids.

Endocannabinoids \& analogs
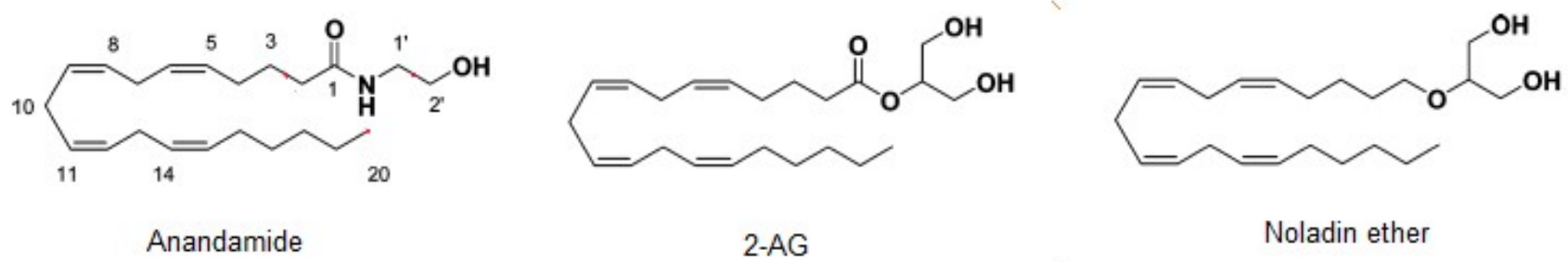

Phytocannabinoids \& derivatives

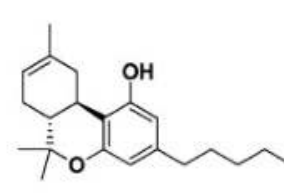

$\Delta^{8}$-THC

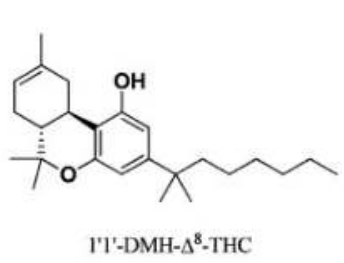

Synthetic cannabinoids

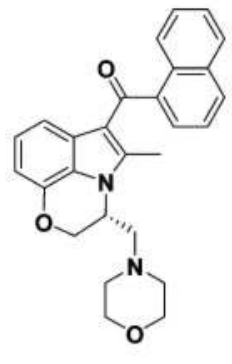

WIN 55212-2
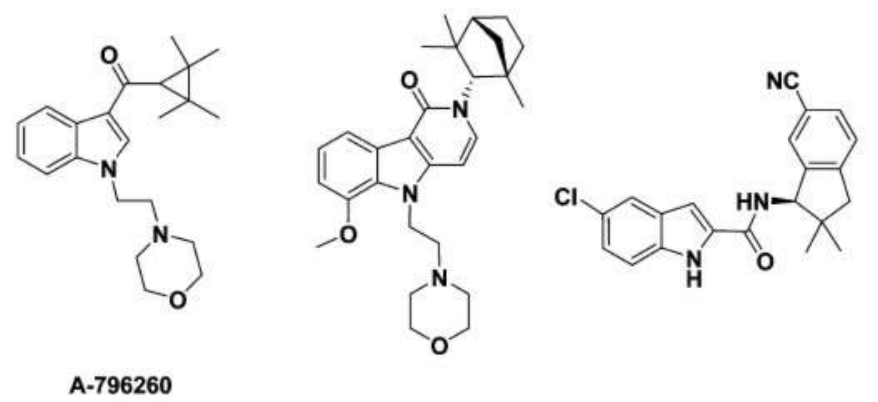
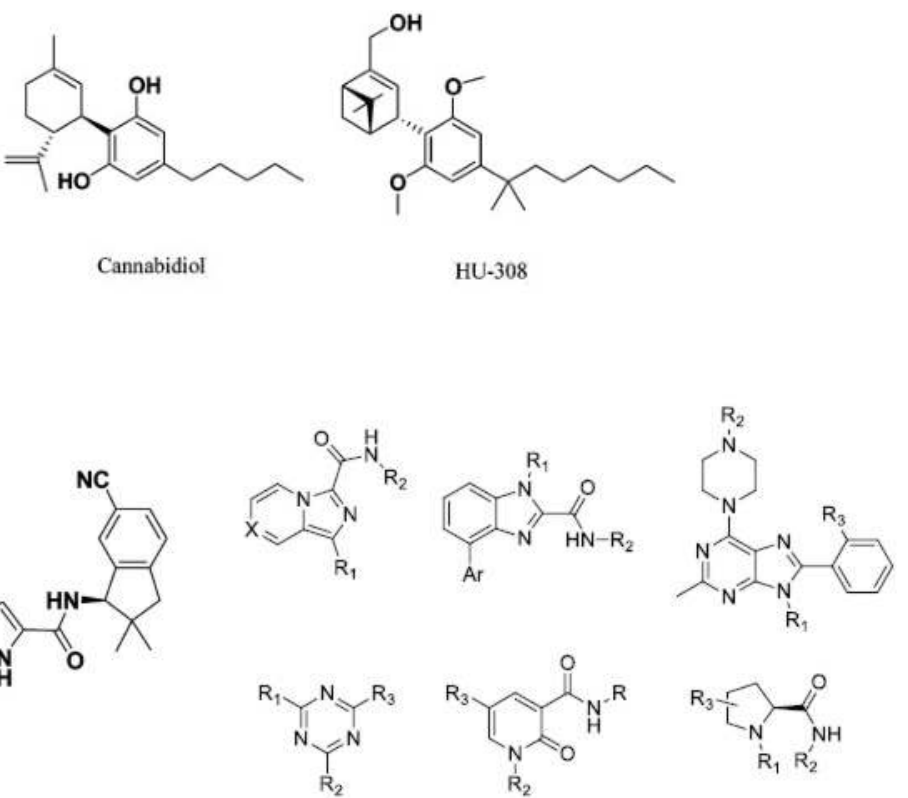

Cannabidiol

HU-308 


\section{High structural diversity of synthetic cannabinoids}
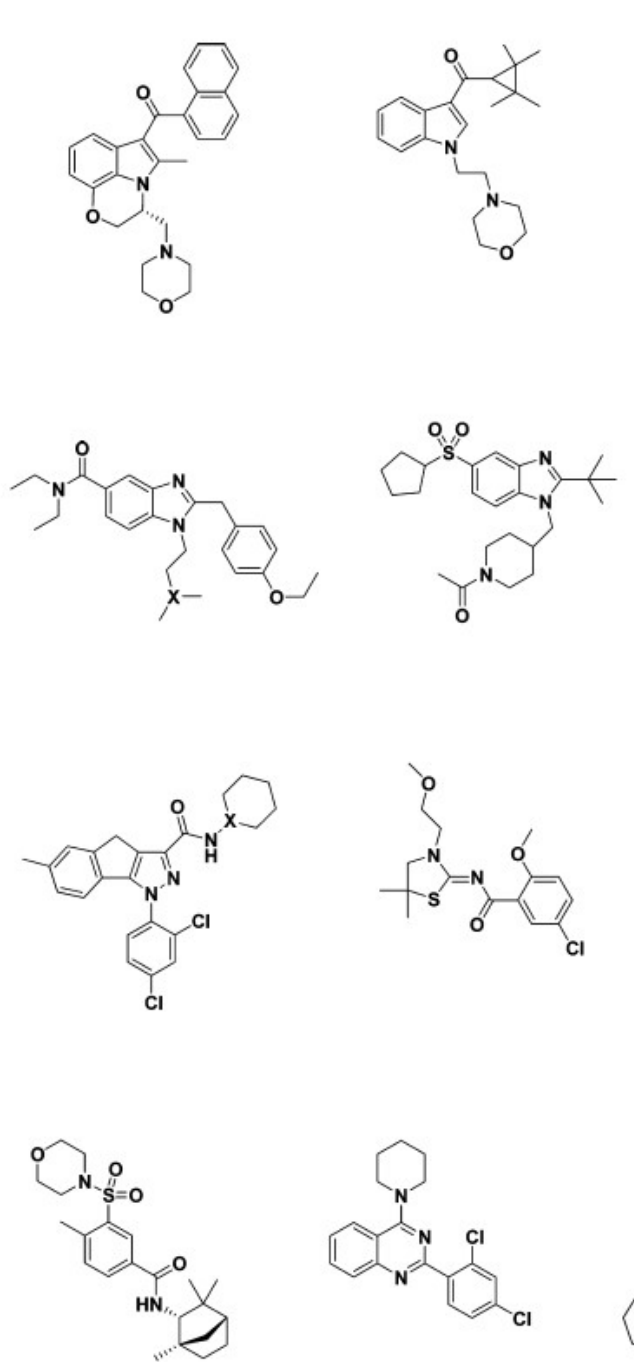
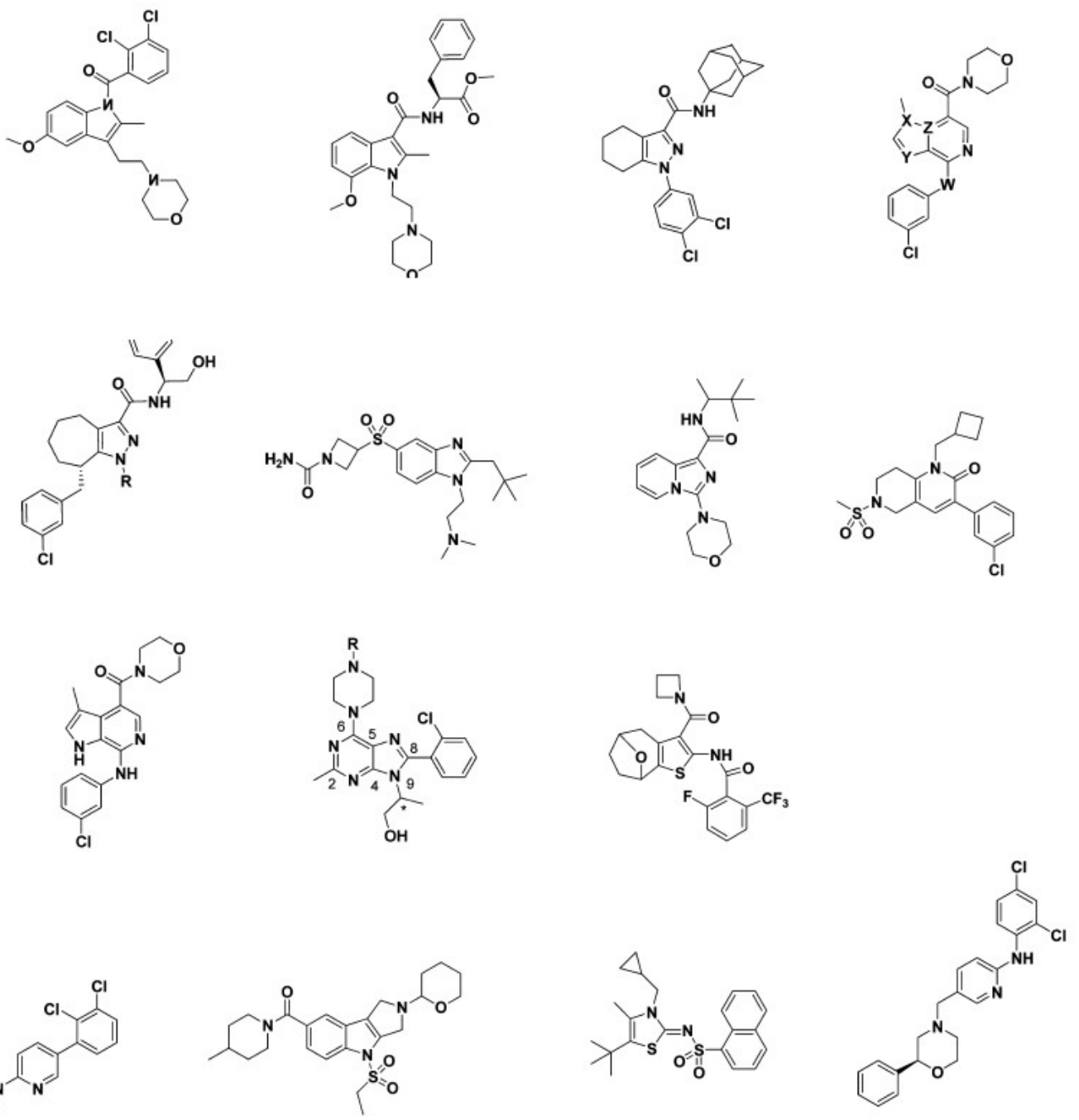

Han, S.; Thatte, J.; Buzard, D. J.; Jones, R. M., Therapeutic utility of cannabinoid receptor type 2 (CB(2)) selective agonists. J Med Chem 2013, 56, (21), 8224-56. 


\section{Results and Discussion}

1) Basic strategy for the CB-ligand template construction (with CB2- or nonselective CB-ligands as building blocks)

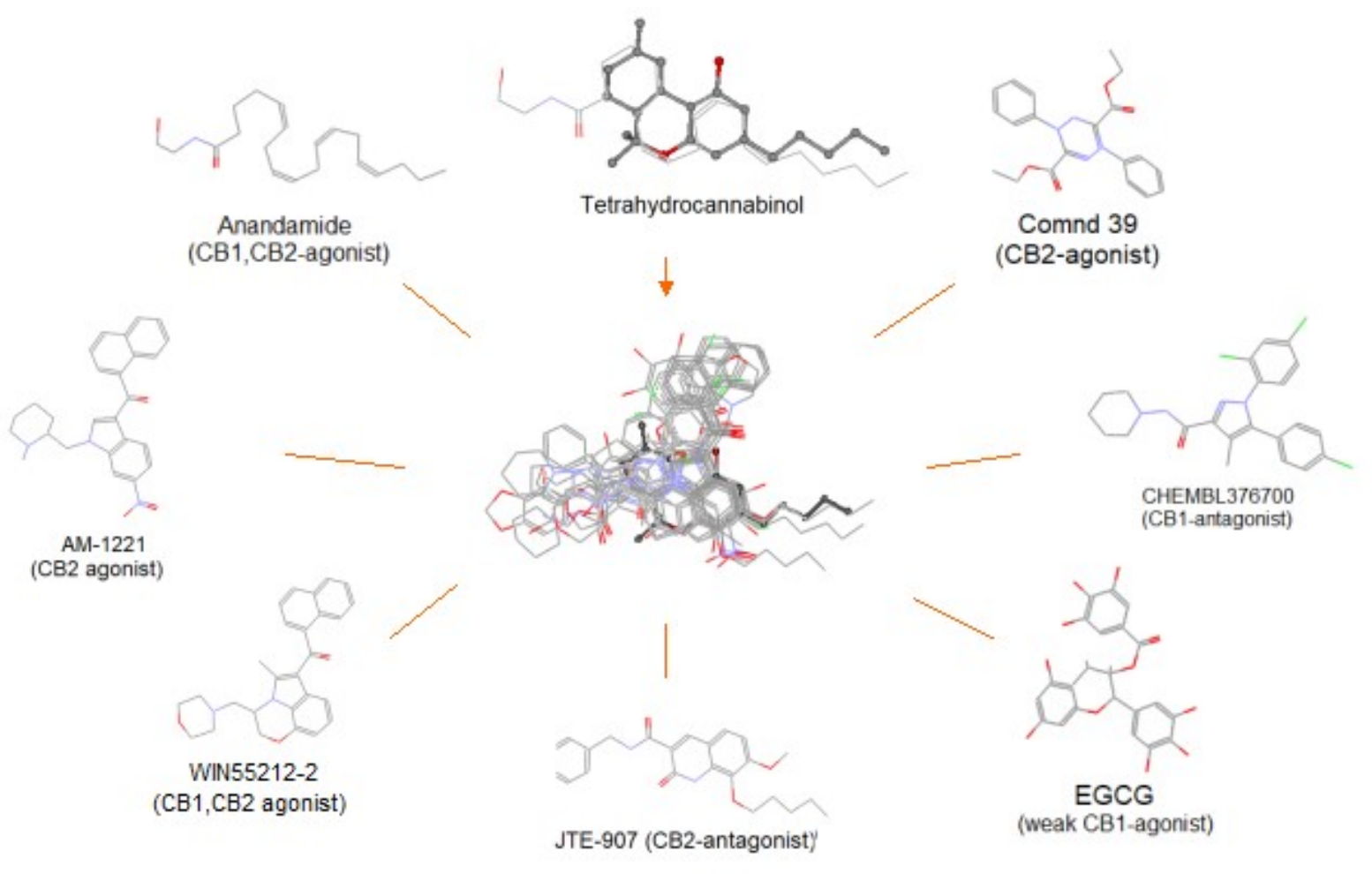

However, the initial trying was hampered, due to the lipid-like nature of CB ligands - most of the scaffolds were flat or flexible, which made hard to validate the lilgand alignment. 


\section{2) Assisted with the crystal structures of $C B$ ligands}

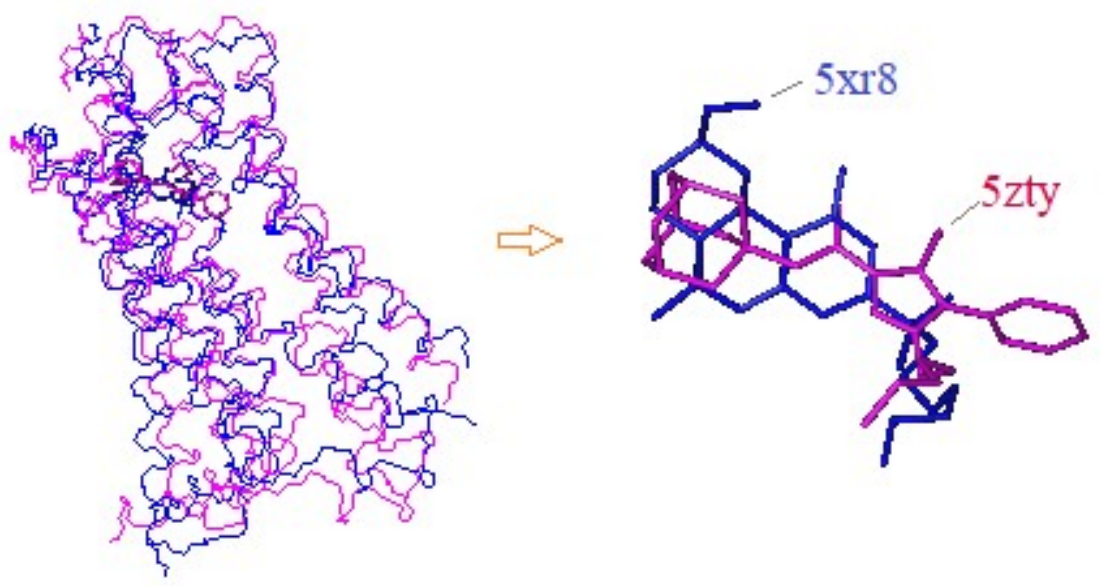

The binding conformations as revealed with the crystal structures of CB ligands provided critical information for the structural correlations between phytoand syntethic cannabinoids, which in turn helped for the construction of the CB-ligand template.

Features of the template:

- A flat and rigid scaffold with a 5-C aliphatic side chain perpendicular to the template scaffold - A/B rings: for alignment of the major scaffolds of most of synthetic CB-ligands

- $\mathrm{C} / \mathrm{D} / \mathrm{E}$ rings: for alignment of the major scaffolds of phytocannabinoids

- A1 ring: for certain $\mathrm{CB}$ antagonists.

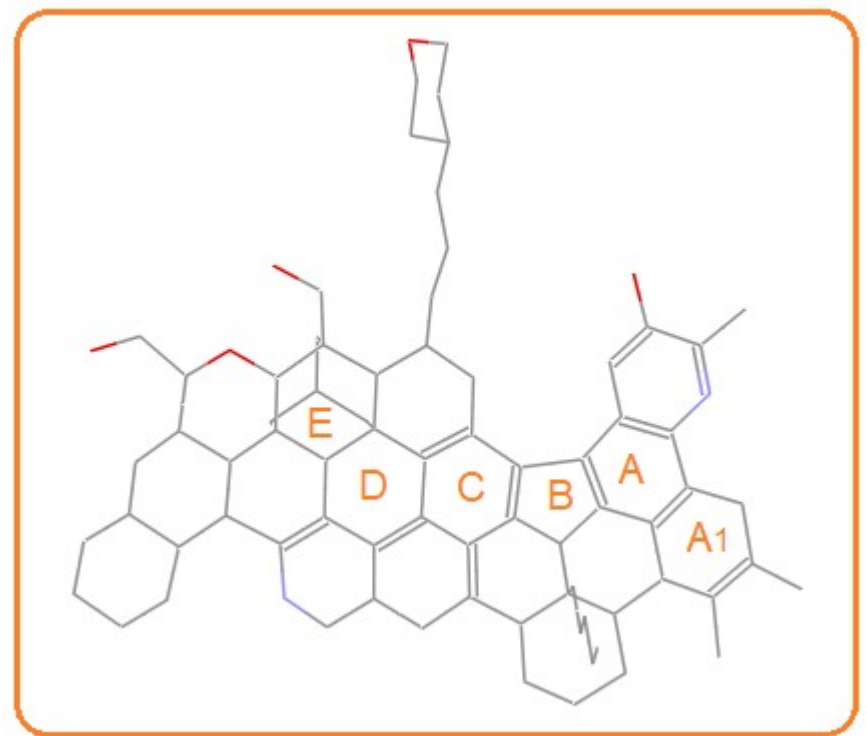




\section{3) Alignment for structural correlations}

\section{Phytocannabinoids}

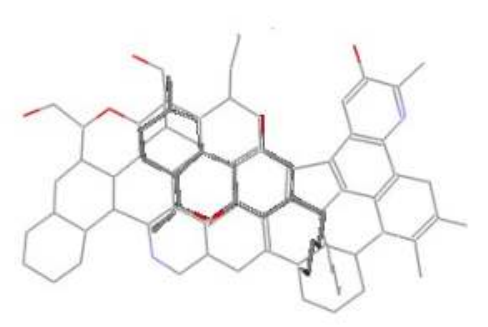

Tetrahydrocannabinol

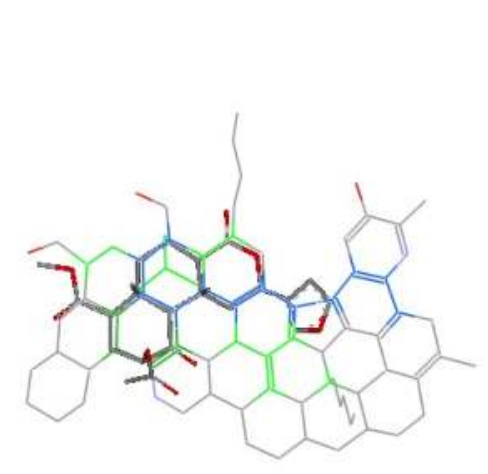

Savinorin $A$ (CB1-ago)

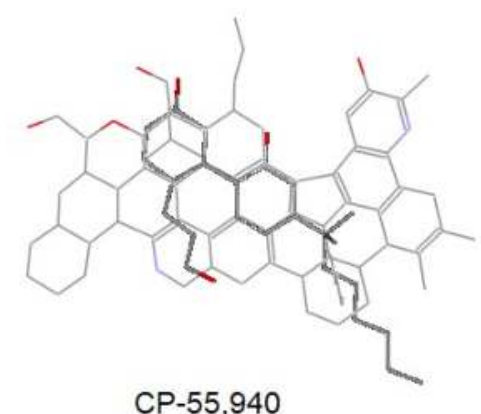

CP- 55,940

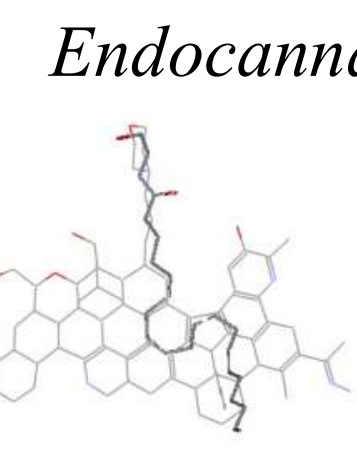

Anandamide $(r 1 / 2=0.25)$

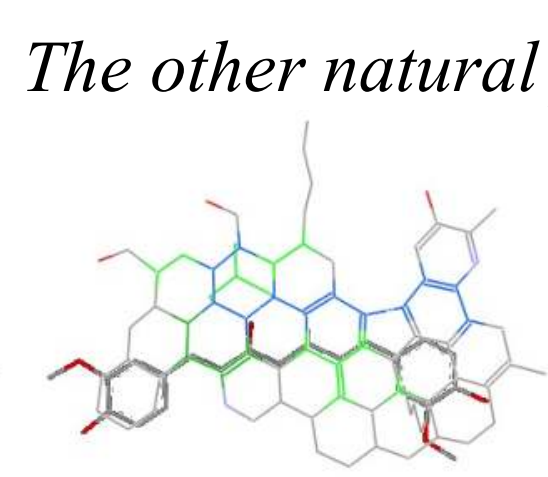

Curcumin (CB1-antg/CB2-ago)

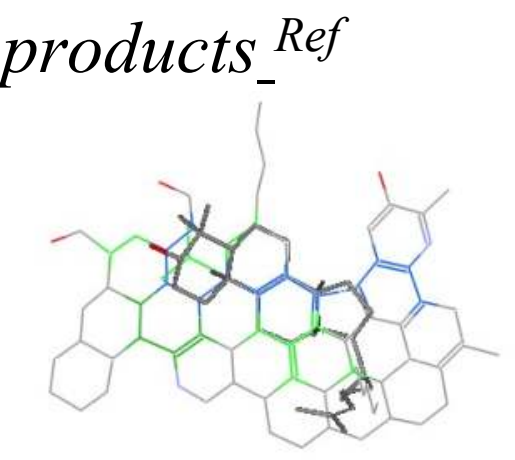

Euphol (CB1/2-ago)

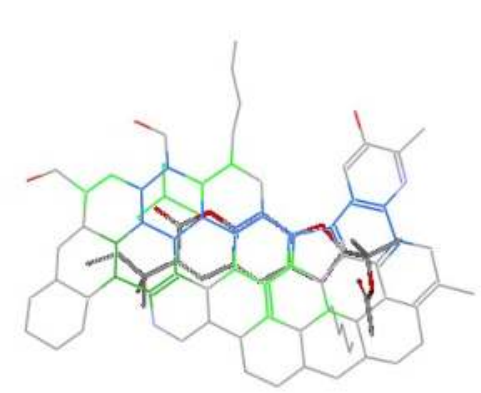

Rutamarin (CB2-ligand)

Ref. Gertsch, J.; Pertwee, R. G.; Di Marzo, V., Phytocannabinoids beyond the Cannabis plant - do they exist? Br J Pharmacol 2010, 160, (3), 523-9. 


\section{Alignment of synthetic cannabinoids}

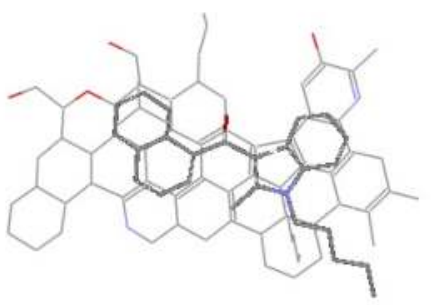

CHEMBL306529

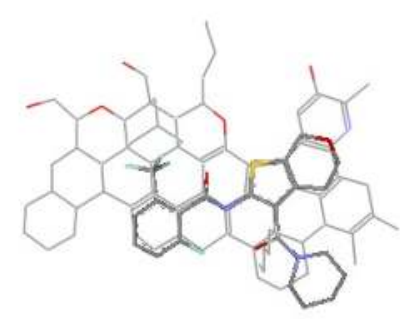

BDBM50402874

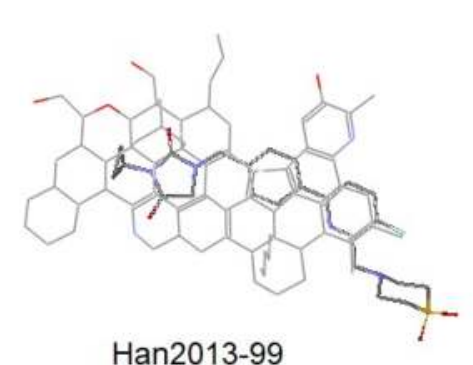

Han2013-99

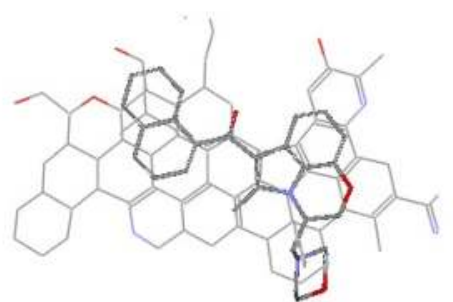

Win55212-2

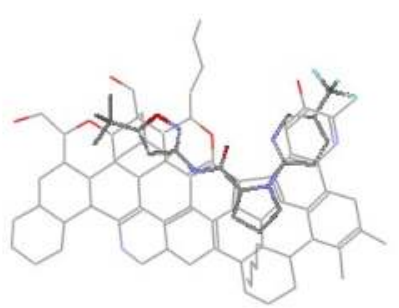

Spinelli2017-6

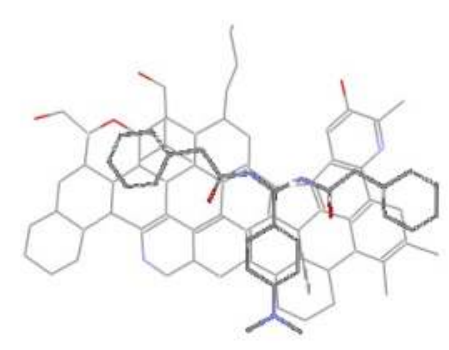

Morales2016-38

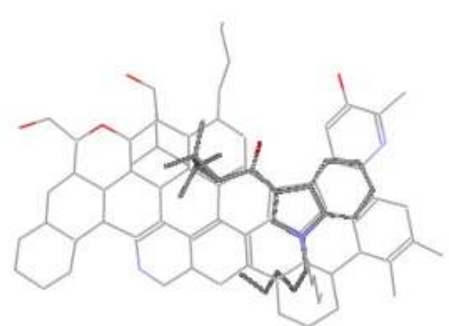

UR-144

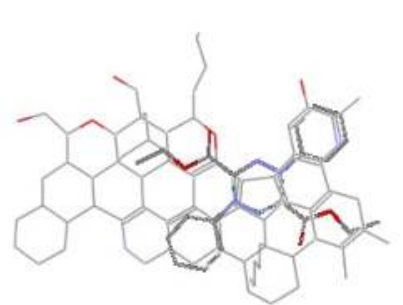

Ashton2008-39

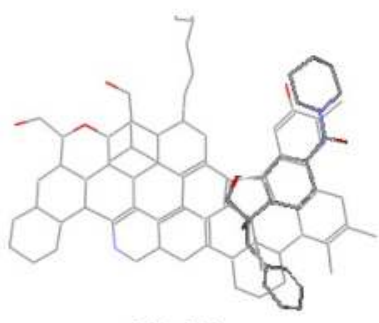

MDA7

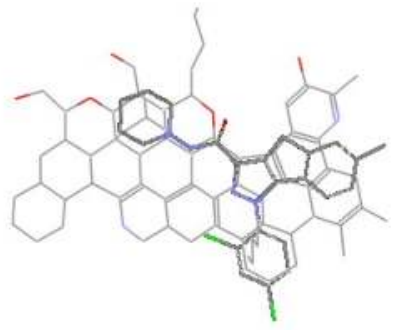

Ashton2008-38

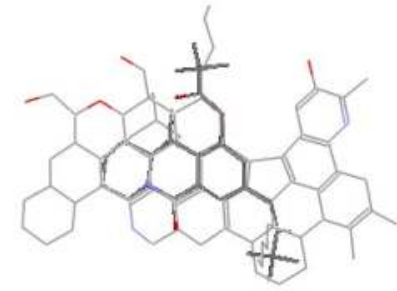

,Ashton2008-41

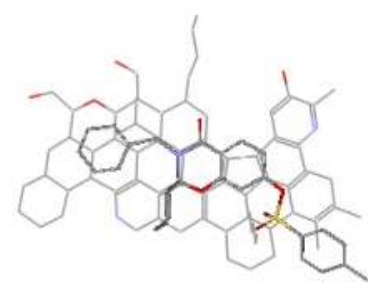

Morales2016-32

(Note: as the template is still in refinement process, most of the current alignments are putative and will need further validation). 


\section{4) SARs interpretation}

Two major alignment patterns were revealed by TAM modeling, which in turn helped to address the basic issues regarding the structural correlations of CB ligands: (1) how phyto- and synthetic-CB ligands are correlated; (2) how the diverse CB ligands are correlated.

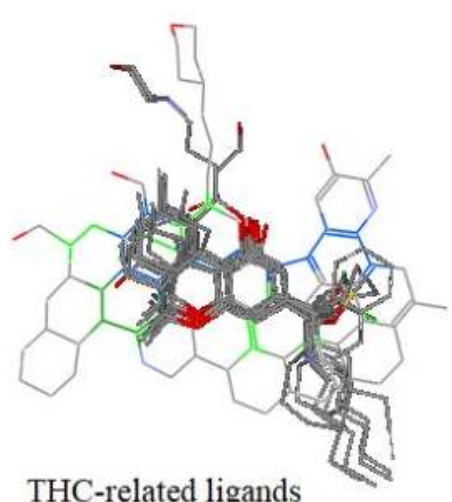

THC-related ligands

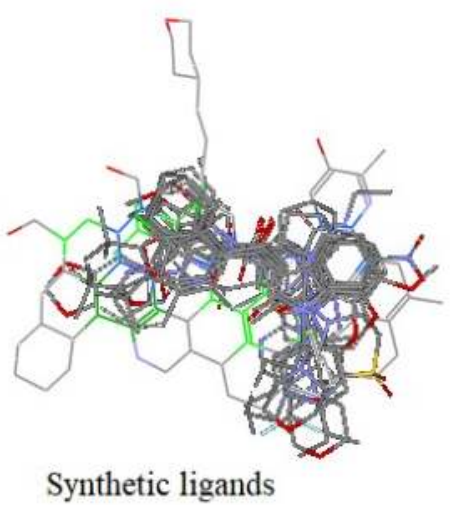

(1)

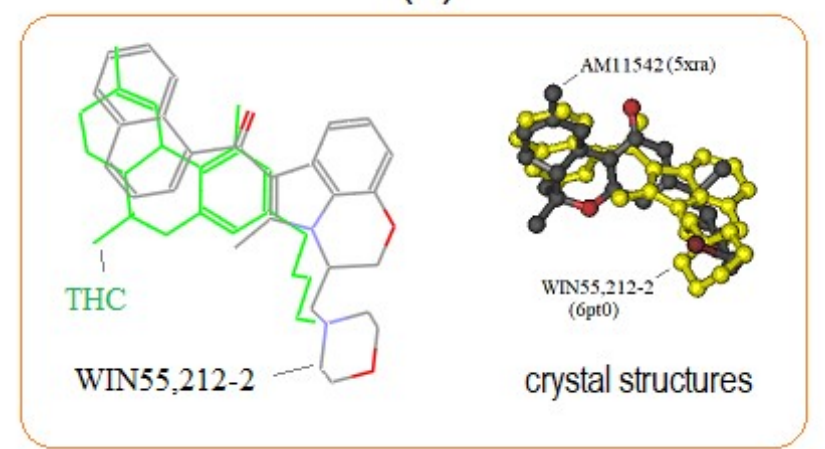

(2)
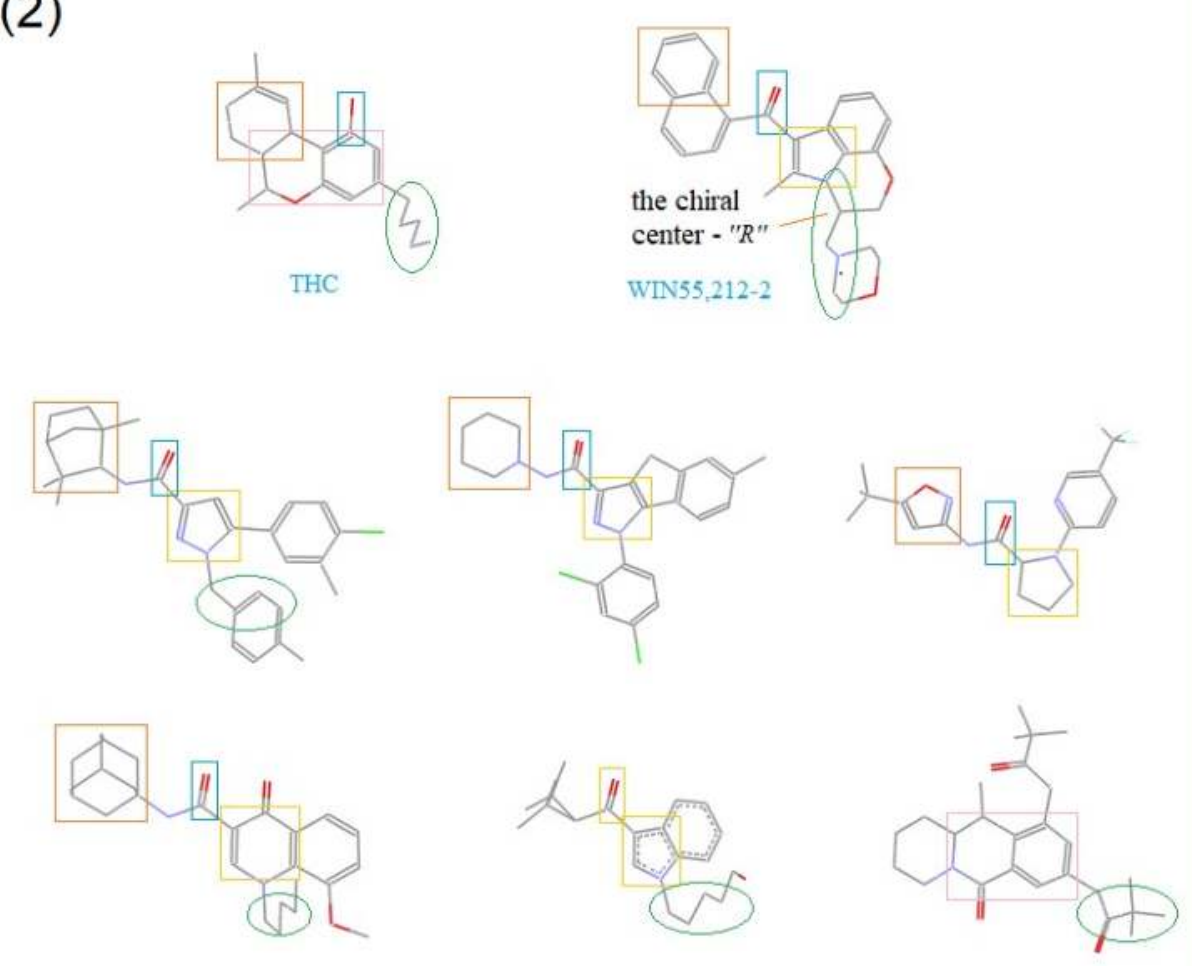


\section{5) The CB2-binding features}

(...maybe scaffold-dependent and still in validation)

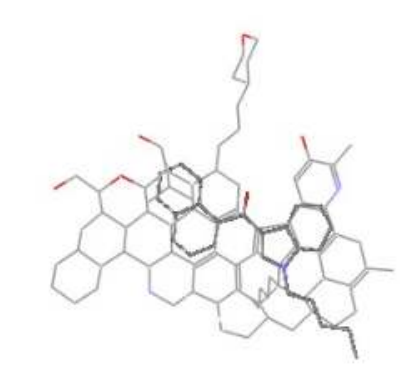

JWH-18 (CB2/1=3/9 nM)

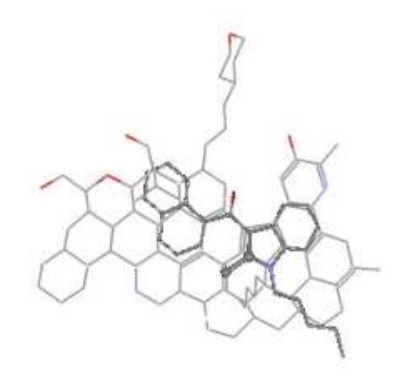

JWH-004 (CB2/1=4/48 nM)

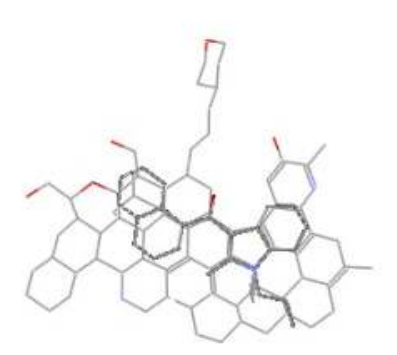

JWH-015 (CB2/1=14/383 nM)
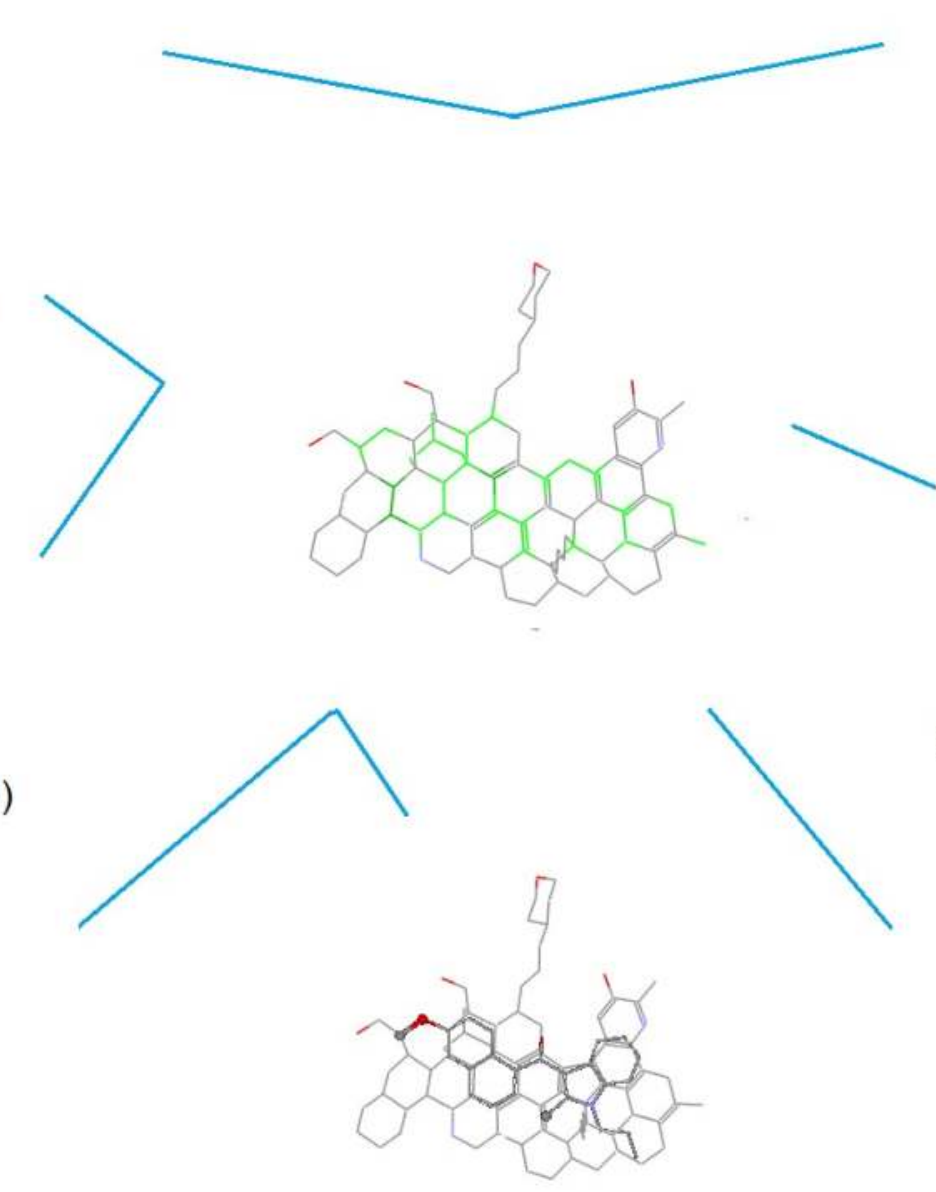

JWH-151 (CB2/1=30/10000 nM)
Han2013 (CB2/1=1.8/10000 nM)
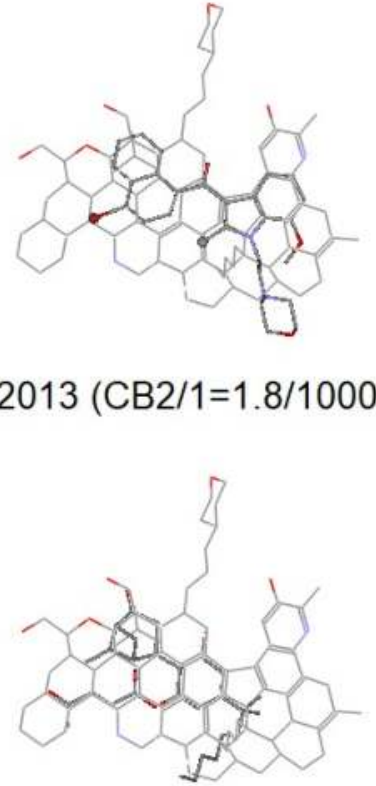

PRS-211375 (CB2/1=17/5585 nM)

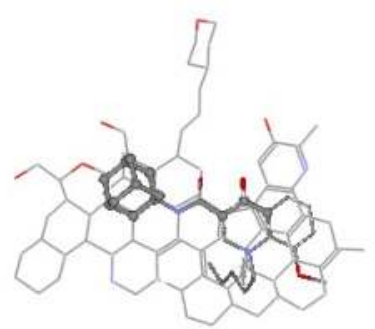

BDBM50350151 CB2/1 $=0.6 / 10000 \mathrm{nM}$ ) 


\section{6) CB-antagonists/Inverse-agonists}

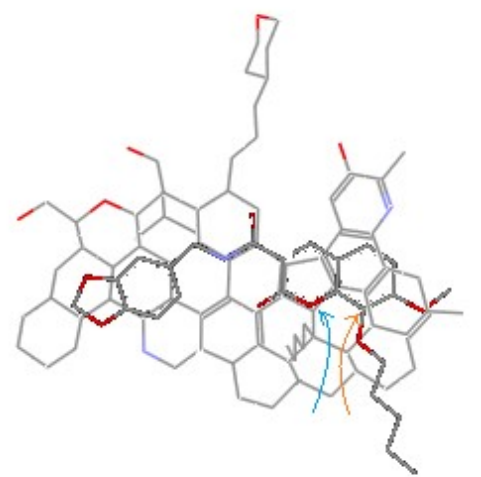

JTE-907 (CB2 inv-ago)

Ref-1

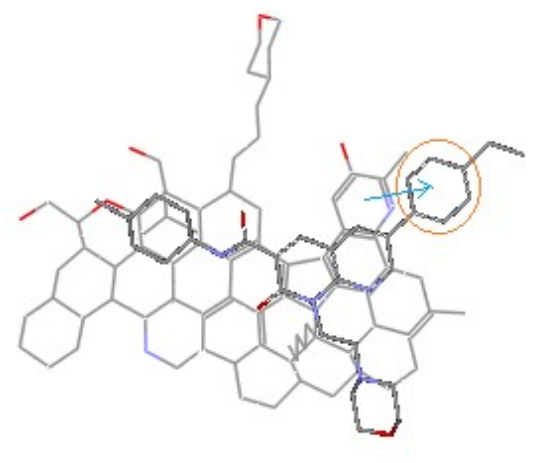

Lucchesi2014-23 (CB2-inv ago)

Ref-2

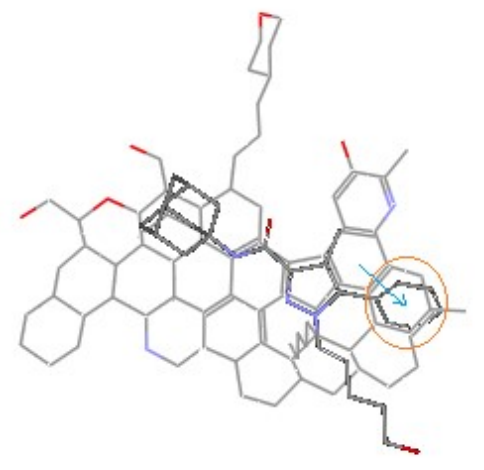

AM10257 (CB2-antg)

Ref-3
Ref-4

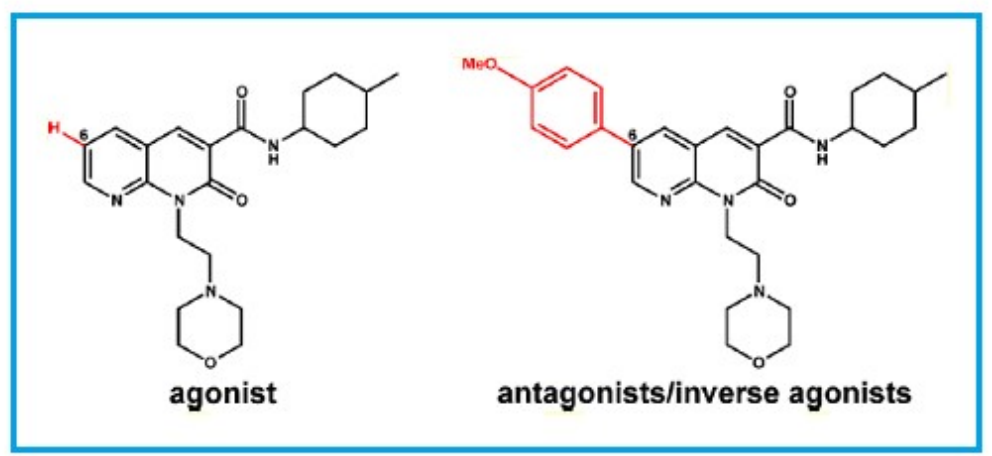

Ref-5

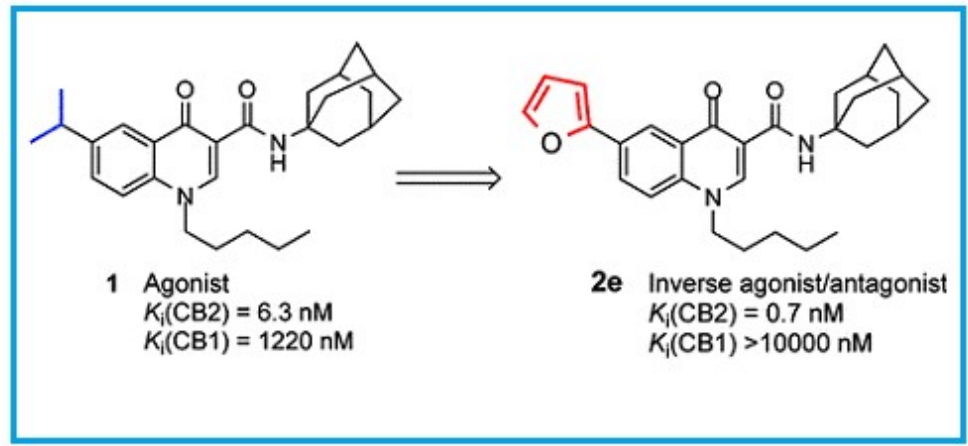

Ref-1: Iwamura, H.; et at., The Journal of pharmacology and experimental therapeutics 2001, 296, (2), 420425. Ref-2. Lucchesi, V.; et at., J Med Chem 2014, 57, (21), 8777-91. Ref-3. Li, X.; et al., Cell 2019, 176, (3), 459-467 e13; Ref-4: Lucchesi, V.; et al., J Med Chem 2014, 57, (21), 8777-91; Ref-5: Pasquini, S. et al., J Med Chem 2010, 53, (16), 5915-28. 


\section{Conclusions}

1. A preliminary template of CB-ligand was constructed based on the innovative template-alignment modeling (TAM).

2. TAM helped for the structural correlations as well as the SAR interpretations of various CB ligands.

3. Continued template refinement is in progress in order to further define the structural features with respect to the CB2-binding selectivity and the antagonism, as well as the more complete ligand binding space, of the template.

\section{Acknowledgements}

Professor Victor Hruby, University of Arizona

Professor Spencer Knapp, Rutgers University 